\title{
Etiological and Clinical Patterns of Isolated Hepatomegaly at Rajshahi, Bangladesh
}

Chanchal Kumar Ghosh, Fakhrul Islam, Ershaduddin Ahmed, Dilip Kumar Ghosh, Azizul Haque, QT Islam, MA Zahir

\begin{abstract}
Background: Hepatomegaly, a common clinical finding, is usually associated with splenomegaly. However, sometimes there may be isolated hepatomegaly without splenomegaly in different pathological conditions. An account of that at Rajshahi, Bangladesh has been given in this study.
\end{abstract}

Materials and methods: This prospective study was carried out in different medicine units of Rajshahi Medical College Hospital, Rajshahi, Bangladesh on consecutive 100 hospitaladmitted patients having isolated hepatomegaly.

Results: Most common cause of isolated hepatomegaly was liver abscess $(34.0 \%)$ followed by congestive cardiac failure $(30.0 \%)$. Viral hepatitis accounted for about $14 \%$ cases of isolated hepatomegaly. Fatty liver and hepatocellular carcinoma were also responsible for isolated hepatomegaly in some patients.

Conclusion: Taken together, isolated hepatomegaly varies from comparatively benign condition like fatty liver to fatal condition like hepatocellular carcinoma. Multicenter study with large sample size is warranted to develop more insights about diagnosis and management of isolated hepatomegaly.

Abbreviations: CCF: Congestive cardiac failure; $\mathrm{RMCH}$ : Rajshahi Medical College and Hospital; HCC: Hepatocellular carcinoma; IHD: Ischemic heart disease; BCS: Budd-Chiari syndrome; HAV: Hepatitis A virus; HBV: Hepatitis B virus; HCV: Hepatitis C virus; HEV: Hepatitis E virus.

Keywords: Isolated hepatomegaly, Prospective study, Liver abscess, Congestive heart failure.

How to cite this article: Ghosh CK, Islam F, Ahmed E, Ghosh DK, Haque A, Islam QT, Zahir MA. Etiological and Clinical Patterns of Isolated Hepatomegaly at Rajshahi, Bangladesh. Euroasian J Hepato-Gastroenterol 2012;2(1):1-4.

\section{Source of support: Nil}

Conflict of interest: None

\section{INTRODUCTION}

The liver is one of the vital organs of the body and plays critical role during synthesis, metabolism, excretion, detoxification and immunity. ${ }^{1-3}$ Although it is only $1 / 40$ th of adult body weight, it receives 1/4th of total circulating blood. This preferential vascular organ also receives exceptional types of blood supply; the liver receives systematic blood through hepatic artery and venous blood from the gut through portal vein. This allows the liver to interact with various noxious materials, food products, and drug metabolites that are carried into the liver through portal circulation. ${ }^{4,5}$ Thus, inflammation, infection and malignant transformation of liver may happen due to various causes. These pathological factors may also induce hepatomegaly. The common causes of hepatomegaly include hepatitis, liver abscess, congestive cardiac failure (CCF), fatty liver, primary and secondary carcinoma of liver, cystic disease, amylodosis, tuberculosis, typhoid fever, malaria, constrictive pericarditis and cardiac tamponade, Budd-Chiari syndrome, glycogen storage disease, biliary obstruction, Reidel's lobe, low lying diaphragm and normal variant. ${ }^{6-15}$ In many cases of hepatomegaly, inflammation of the liver is associated with that of the spleen. However, isolated hepatomegaly may also occur in various pathological conditions. Indeed, almost nothing has been reported about isolated hepatomegaly from Bangladesh.

This study conducted here was accomplished in an academic hospital of the Northern part of Bangladesh. The clinical features of 100 consecutive patients with isolated hepatomegaly were evaluated. In addition, clinical profiles of the patients and demographic data were collected. This study would provide insights about diagnosis and management of this important pathological condition in clinical medicine.

\section{MATERIALS AND METHODS}

The present prospective study was carried out in different medicine units of Rajshahi Medical College Hospital (RMCH), Rajshahi, Bangladesh on consecutive 100 admitted patients having isolated hepatomegaly. Isolated hepatomegaly was considered when hepatomegaly was not associated with splenomegaly; clinically and sonologically. Hepatomegaly was considered when the total span of the liver in the midclavicular line as determined by clinical examination (palpation and percussion) was $>10 \mathrm{~cm}$ and $>12.5 \mathrm{~cm}$ by sonography. After the preliminary selection of patients, detailed clinical history, thorough physical examination and laboratory investigations were done. The details were recorded according to predesigned questionnaire. Investigations, such as complete blood count and erythrocyte sedimentation rate, routine and microscopic examination of stool and urine, serum bilirubin, serum glutamic transferase, serum alkaline phosphatase, ultrasonogram of whole abdomen were performed in all patients. Other investigations like hepatitis B surface antigen, antibody to hepatitis $\mathrm{C}$ virus (HCV), prothrombin 
time, alpha fetoprotein, chest X-ray, aspiration of pus for analytic study (physical examination, cytology, staining, culture and sensitivity), CT scan, electrocardiogram, and liver biopsy were done in selected cases. Aspiration of liver abscess was done under sonographic guidance in Nuclear Medicine Department of RMCH. Aspirated materials were sent for microscopical examination and culture and sensitivity in the Department of Microbiology, RMCH. Liver biopsy was done in selected patients using Tru-cut needle and the histopathological examinations were done in the Department of Pathology, RMCH.

\section{RESULTS}

Total one hundred consecutive patients with isolated hepatomegaly were included in the study. The mean age of the patients was 42.2 years (standard deviation, 3.2 years) with a range of 15 to 80 years. The predominant age group was between 41 and 50 years $(29.0 \%)$, followed by the age group of 31 to 40 years $(24.0 \%)$ and 21 to 30 years $(20.0 \%)$. Among them $76.0 \%$ were male and $24.0 \%$ were female. Most common cause of isolated hepatomegaly in the study population was liver abscess $(34.0 \%)$ followed by CCF $(30.0 \%)$ and viral hepatitis $(14.0 \%)$, secondary carcinoma of liver $(8.0 \%)$ and primary HCC $(6.0 \%)$. Other causes of isolated hepatomegaly were hydatid cyst of liver $(2.0 \%)$, fatty liver $(2.0 \%)$, Budd-Chiari syndrome (1.0\%), autoimmune hepatitis $(1.0 \%)$, tuberculosis $(1.0 \%)$ and typhoid fever (1.0\%) (Fig. 1). Out of 34 patients with liver abscesses, 20 had amebic abscesses and abscess in 14 were due to pyogenic causes. A single abscess focus was seen in 16 patients, whereas, multiple abscesses were detected in 18 patients.

Among 30 patients with CCF, cor pulmonale (40.0\%) was the commonest cause of isolated hepatomegaly, followed by valvular heart disease $(26.7 \%)$, ischemic heart

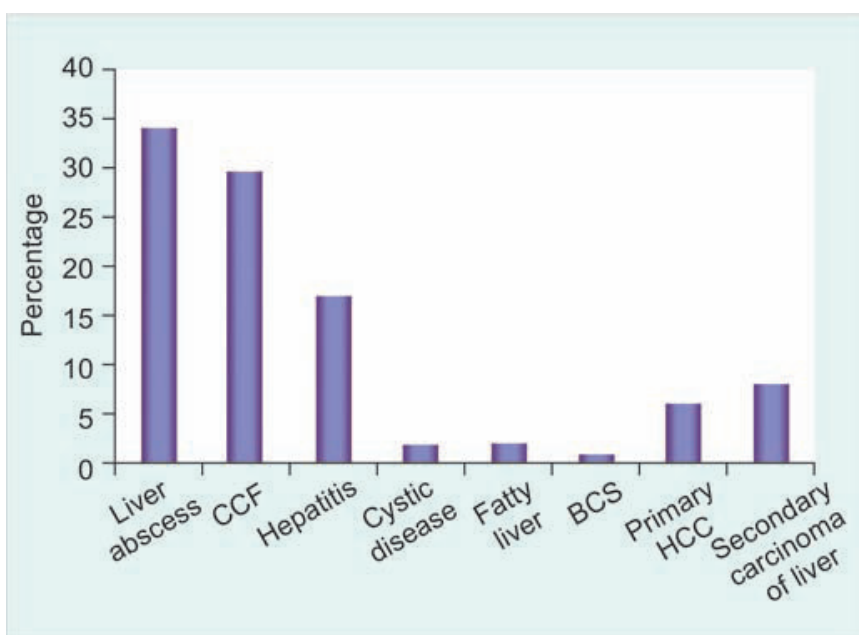

Fig. 1: Causes of isolated hepatomegaly at Bangladesh. CCF, congestive heart failure, BCS diseases $(10.0 \%)$ and cardiomyopathy (10.0\%). Other causes of CCF in the present study were constrictive pericarditis $(6.7 \%)$, hypertensive heart disease $(3.3 \%)$ and congenital heart disease (3.3\%).

Among the 17 patients with hepatitis, the main cause of hepatitis was hepatitis virus $(82.4 \%)$. Hepatitis due to hepatitis A virus (HAV), hepatitis B virus (HBV), HCV and hepatitis E virus (HEV) were 35.3, 23.4, 11.8 and 5.9\% respectively. Other causes of hepatitis were dengue virus, tuberculosis, typhoid and autoimmune diseases (5.9\% each). Among 14 patients of malignancy of the liver, six patients $(42.86 \%)$ were diagnosed as primary hepatocellular carcinoma and eight (57.14\%) were secondary carcinoma of liver. Abdominal pain (80.0\%), loss of appetite (76.0\%), general weakness $(60.0 \%)$ and fever $(54.0 \%)$ were the common clinical presentations of these patients. Other clinical presentations were loss of weight $(51.0 \%)$, pallor $(51.0 \%)$, nausea and vomiting $(44.0 \%)$, jaundice $(42.0 \%)$ and exertional breathlessness $(31.0 \%)$.

Among the total cases $83.0 \%$ patients had tender hepatomegaly and $17.0 \%$ did not have any complain of pain. The consistency of the liver was soft in $48.0 \%$ patients, firm in $36 \%$ patients and hard in $16 \%$ patients. Among the primary $\mathrm{HCC}, 66.7 \%$ were associated with $\mathrm{HBV}$ infection and $33.3 \%$ were anti-HCV positive.

Liver span was measured by ultrasound. Liver was markedly enlarged in case of carcinoma of both primary and secondary $(64.3 \%)$, whereas, the liver was slightly enlarged in most cases with hepatitis (64.7\%) (Table 1).

Liver biopsy was done in 25 selected patients. Among them, histopathological findings of eight (32\%) were consistent with secondary carcinoma of liver, six $(24.0 \%)$ were primary HCC, three $(12.0 \%)$ were liver abscess, two $(8.0 \%)$ fatty liver, one $(4.0 \%)$ tubercular granuloma and inconclusive findings were seen in five cases $(20.0 \%)$ (Table 2).

\section{DISCUSSION}

The aim of this study was to find out the etiology and clinical pattern of isolated hepatomegaly among the patients admitted in different medicine units of RMCH. In this study hepatomegaly was defined when the span of the liver was above the upper limit of normal. There was a male predominancy in patients with isolated hepatomegaly, which support what Khan et al have reported about male predominance about hepatomegaly. ${ }^{6}$

The most common cause of isolated hepatomegaly was liver abscess followed by $\mathrm{CCF}$, hepatitis, primary hepatocellular carcinoma, secondary carcinoma of liver, cystic diseases of liver, fatty change and Budd-Chiari 


\begin{tabular}{lcccc}
\hline \multicolumn{2}{c}{ Table 1: The extent of isolated hepatomegaly in different pathological conditions } \\
\hline & \multicolumn{3}{c}{ Liver span (in cm) } & Total \\
\cline { 2 - 4 } Disease & $12.5-15.5$ & $15.6-18.5$ & $>18.6$ & $34(100)$ \\
& $7(20.6)$ & $15(44.1)$ & $12(35.3)$ & $30(100)$ \\
Liver abscess & $18(60.0)$ & $10(33.3)$ & $2(6.7)$ & $17(100)$ \\
Congestive cardiac failure & $11(64.7)$ & $6(35.3)$ & $9(0.0)$ & $14(100)$ \\
Carcinoma of the liver & $0(0.0)$ & $5(35.7)$ & $1(20.0)$ & $05(100)$ \\
Others & $1(20.0)$ & $3(60.0)$ & & $9(3)$ \\
\hline
\end{tabular}

Values in the parenthesis indicate percentage

Table 2: Histopathological diagnosis of liver biopsy of patients with isolated hepatomegaly

\begin{tabular}{lcc}
\hline Histopathological diagnosis & Frequency & Percentage \\
\hline Primary HCC & 6 & 24.0 \\
Secondary cancer of liver & 8 & 32.0 \\
Tubercular hepatitis & 1 & 04.0 \\
Consistent with liver abscess & 3 & 12.0 \\
Fatty liver & 2 & 08.0 \\
Normal histology & 5 & 20.0 \\
\hline Total & 25 & 100.0 \\
\hline
\end{tabular}

Liver biopsy was performed in 25 patients with isolated hepatomegaly

syndrome. There is no study, so far in our country showing the etiologies and clinical profiles of isolated hepatomegaly and thus a comparative analysis could not be accomplished. But, some findings of our study are comparable with those of other countries. ${ }^{6-15}$ Shennak et al have shown CCF $(38.5 \%)$, carcinoma of liver $(19.6 \%)$, acute hepatitis $(13.5 \%)$, cystic diseases $(7.88 \%)$, carcinoma of liver (19.6\%), fatty change $(5.6 \%)$, and liver abscesses $(2.4 \%)$ as major causes of isolated hepatomegaly from a cohort of 800 patients from Jordan. ${ }^{7}$ In the present study, the higher incidence of liver abscesses have been contributed by social and public health factors like; lower socioeconomic condition, malnutrition, poor sanitary condition, contact infection of E. histolytica, consumption of local wine prepared by partially fermentation of palm juice. The commonest cause of liver abscess worldwide is amebiasis, but in the developed world pyogenic causes are of increasing importance. ${ }^{1}$ In the present study out of 34 patients with liver abscess, $58.8 \%$ were due to amebic abscesses and $41.2 \%$ were due to pyogenic abscess.

We found cor pulmonale as the commonest cause of $\mathrm{CCF}$, followed by valvular heart disease, ischemic heart disease and cardiomyopathy. The incidence of CCF is on increase due to poorly-developed health care delivery system and late attendance of patients to physicians for social reasons.

Hepatitis due to HAV, HBV, HCV, HEV and others etiologies were prevalent among patients with isolated hepatomegaly. Dengue is an emerging and serious public health problem at Bangladesh. Assessment of liver size and their function may help to accomplish early proper diagnosis of dengu.

HCC is a primary malignancy of the liver. It is a major complication of hepatitis virus infections in many instances, hepatomegaly is the first subjective symptom of HCC in Bangladesh, as most of the advanced cases of HCC attend physicians. This tremendously restricts treatment option of HCC patients in clinics.

In this study two patients with isolated hepatomegaly had fatty change of liver; one was due to alcohol and the other patient had clinical feature suggestive of malnutrition. Two patients presented with hydatid cysts which was diagnosed by enlarged right lobe of liver with cystic consistency, sonologic findings and by ELISA test. Hydatid cyst is a zoonotic disease with an endemic regional distribution and the most common localization is the liver (50-70\% of all cases). One patient in this study diagnosed as Budd-Chiari syndrome, presented with rapid appearance of huge hepatomegaly, ascites with collateral veins in anterior abdominal wall.

Taken together, the present report a clinical study that assessed causes and clinical factors related to isolated hepatomegaly in Bangladesh. Although Bangladesh is a small country, it possesses variable geographical characteristics and people with different social nature. In this study, analyzes were done in 100 patients with isolated hepatomegaly. If similar studies are done in other parts of the country, data will be accumulated to draw a profile of isolated hepatomegaly in different parts of this country. It is highly tempting to consider that different etiological factors would be prevalent in different parts of Bangladesh on the basis of local social and health care delivery system. Patients presented with isolated hepatomegaly vary from very benign condition like fatty liver to fatal condition as hepatocellular carcinoma. In the present study it was found that liver abscess is the commonest cause of isolated hepatomegaly followed by congestive cardiac failure.

\section{REFERENCES}

1. Chapman RW, Collier JD, Hayes PC. Liver and biliary tract disease. In Davidson's principles and practice of medicine 
(20th ed). In: Boon NA, Colledge NR, Walker BR, Hunter JAA (Eds). Edinburgh, Churchill Livingstone, 2006;935-89.

2. Sherlock S, Doobey J. Diseases of the liver and biliary system (10th ed). London; Blackwell Science 1997.

3. Kumer P, Clark M. Liver, biliary tract and pancreatic disease. Clinical medicine (5th ed). Edinburgh: WB Saunders 2002; 335-404.

4. Mehal WZ, Azzaroli F, Crispe IN. Immunology of the healthy liver: Old questions and new insights. Gastroenterology 2001; 120:250-60.

5. Crispe IN, Dao T, Klugewitz K, Mehal WZ, Metz DP. The liver as a site of T-cell apoptosis: Graveyard or killing field? Immunol Rev 2000;174:47-62.

6. Khan R, Hamid S, Abid S, et al. Predictive factors for early aspiration in liver abscess. World J Gastroenterol 2008;14: 2089-93.

7. Shennak MM, Tarawneh MS, Arm-SS, Al-Sheikh TM, AbuRajab MT, Crec SS. Pattern of hepatomegaly in Jordanians. Ann Trop Med Parasitol 1985;79:443-48.

8. Bosch FX, Ribes J, Diaz M. Primary liver cancer: Worldwide incidence and trends. Gastroenterology 2004;127(5 Suppl 1): S5-16.

9. Aydinli M, Bayraktar Y. Budd-Chiari syndrome: Etiology, pathogenesis and diagnosis. World J Gastroenterol 2007;13: 2693-96.

10. Rahman S, Zaki KMJ, Fazal K. Hydatid disease: An overview. The ORION Medical Journal 2004;17:144-45.

11. Christopher WD, Miguel A. Amebic liver abscess. Southern Medical Journal 2004;97:673-82.

12. Francois R, Normand J, Lafay M, Doucet-Bon J, Gillet P, Chatelain P. Calcified constrictive pericarditis manifested by isolated hepatomegaly. Arch Fr Pediatr 1979;36:696-98.

13. Balsarkar D, Joshi MA. Isolated tuberculous hepatic abscess in a non-immunocompromised patient. J Postgrad Med 2000; 46:108-09.

14. Azad AK, Roy PK, Rahman MT, Hasan M. Prognostic factors of hepatocellular carcinoma (HCC). Bangladesh Journal of Medicine 2002;13:26-28
15. Kocer NE, Kibar Y, Guldur ME, Deniz H, Bakir K. A retrospective study on the coexistence of hydatid cyst and aspergillosis, Int J Infect Dis 2007.

\section{ABOUT THE AUTHORS}

\section{Chanchal Kumar Ghosh (Corresponding Author)}

Department of Gastroenterology, Sir Salimullah Medical College, Dhaka, Bangladesh, Phone: 880-1711855075, e-mail: dr.chanchalghosh@gmail.com

\section{Fakhrul Islam}

Department of Medicine, Holy Family Red Crescent Medical College Dhaka, Bangladesh

\section{Ershaduddin Ahmed}

Department of Gastroenterology, Chittagong Medical College Chittagong, Bangladesh

\section{Dilip Kumar Ghosh}

Department of Gastroenterology, Shaheed Suhrawardy Medical College, Dhaka, Bangladesh

\section{Azizul Haque}

Department of Medicine, Shaheed Ziaur Rahman Medical College Bogra, Bangladesh

\section{QT Islam}

Department of Medicine, Dhaka Medical College, Dhaka, Bangladesh

\section{MA Zahir}

Department of Medicine, Sir Salimullah Medical College, Dhaka Bangladesh 